\title{
The Taida Institute for Mathematical Sciences (TIMS)
}

\author{
by Jing Yu and Ching-Lung Wang \\ Department of Mathematics, National Taiwan University, Taipei
}

Taida Institute for Mathematical Sciences (TIMS) launched its program in August, 2006. It is located at the Mathematics Research Center Building in the Northern campus of National Taiwan University, Taipei, Taiwan. The program was initiated by the academician Chang-Shou Lin, who was also the first director of TIMS and chair professor of Taida, i.e. National Taiwan University. The director now is Jing $\mathrm{Yu}$, also academician and

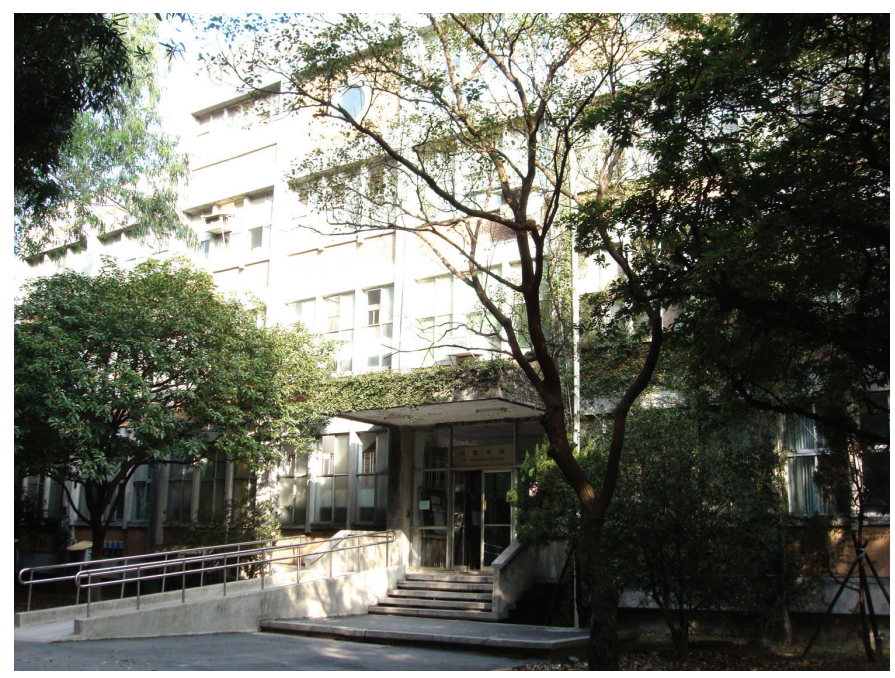

Taida Institute for Mathematical Sciences

chair professor of Taida.

The major goals of TIMS are:

- To be a research center for interdisciplinary and inter-college study of mathematical sciences.

- To be an international mathematical center of knowledge innovation and outstanding research.

- To be a fostering center for mathematical talents.

- To be a communication platform among domestic or oversea academic institutions.

\section{Funding}

Fundings for the operation of TIMS come from Ministry of Education, National Taiwan University, and grants from the National Science Councils. Currently the faculty include: Shu-Cheng Chang, Hung Chen, Chen-Yu Chi, Wu-Yen Chuang, Ming-Lun Hsieh, Chang-Shou Lin, Hui-Wen Lin, Tai-Chia Lin, Chin-Lung Wang, Weichung Wang, Jeng-Daw Yu, Jing Yu. There are also around half-dozen post doctors at TIMS working with the various research groups. In total there are 92 foreign visitors to TIMS during the year 2012.

\section{Projects}

Currently the research directions at TIMS are:

- PDE: Nonlinear Elliptic Equations, Nonlinear Parabolic Equation, Applications to Mathematical Physics, and Mathematical Biology (including computation).

- Geometric Analysis: Geometric Flows, General Relativity.

- Algebraic Geometry: Quantum geometry, Analytic aspects of the minimal model program.

- Number theory: Arithmetic Geometry, Transcendence, Cohomology, Iwasawa Theory, Automorphic Representations.

- Computational Mathematics and applied sciences.

- Statistics: High-dimensional Regression Analysis, Statistical Evaluation of Medical Tests for Classification and Prediction, Statistical Image Analysis.

Major activities designed at TIMS: regular biweekly seminars in each research direction, annual distinguished lecture series, international cooperations with multinational research teams, summer and/or winter schools with short courses organized in each direction, international visitor program, introductory courses designed for 
initiating graduate students into mathematical researches, international workshops focused on selected frontier research topics, international conferences on key topics, and publications of conference proceedings.

International Conferences held at TIMS in 2012 includes: Integrable systems and geometric PDEs, Oct.

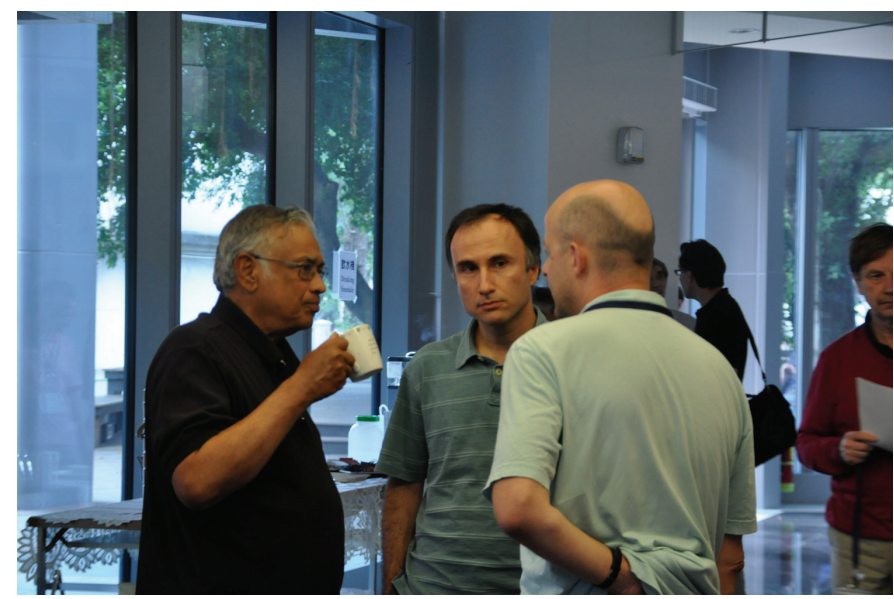

Left to right: S. R. S. Varadhan, Claudio Landim, and Stefano Olla

20-22, the Fourth TIMS-OCAMI joint workshop on differential geometry and geometric analysis Mar. 17-19, the 3rd east Asian number theory conference, Jan. 16-19.

\section{Activities and Achievements}

We make efforts to enlarge the international visibility of TIMS, inviting most distinguished long-term visiting professor from abroad to work together with us, estab-

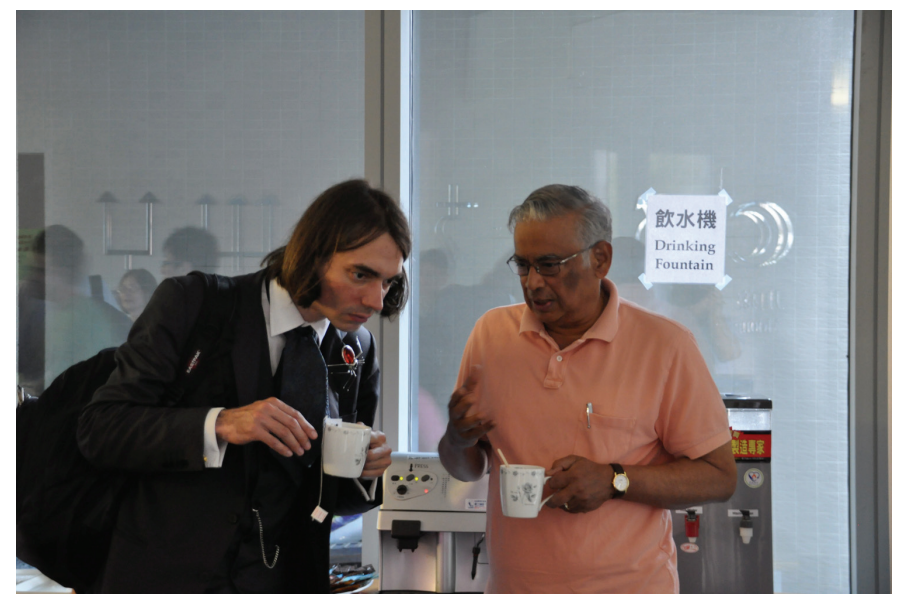

Left to right: Cedric Villani and S. R. S. Varadhan

lishing closer ties with the most prestigious research centers of the world, and publish our works at the top journals. In 2011/12, Shing-Tung Yau of Harvard University, Mu-Tao Wang of Columbia University, and Bong Lian of Brandeis University are the TIMS visiting chair professors. Academician S.-T. Yau's lecture series (49 lectures) is on open problems and discussions of frontier developments.

Recent breakthroughs at TIMS including the following five pieces of deep works:

- $\quad$ Flops, motives and invariance of quantum rings, by Y.-P. Lee, H.-W. Lin, and C.-L. Wang, in Annals of Math. 2010.

- Greens functions, and the mean field equations on tori, by C.-S. Lin and C.-L. Wang, in Annals of Math. 2010.

- $\quad$ Classification and nondegeneracy of SU(n+1) Toda system with singular sources, by D. Y. Wei and C.-S. Lin in Inventiones Math. 2012.

- $\quad$ On the mu-invariant of anticyclotomic $p$-adic $L$-functions for CM fields, by M.-L. Hsieh, in Crelle 2012.

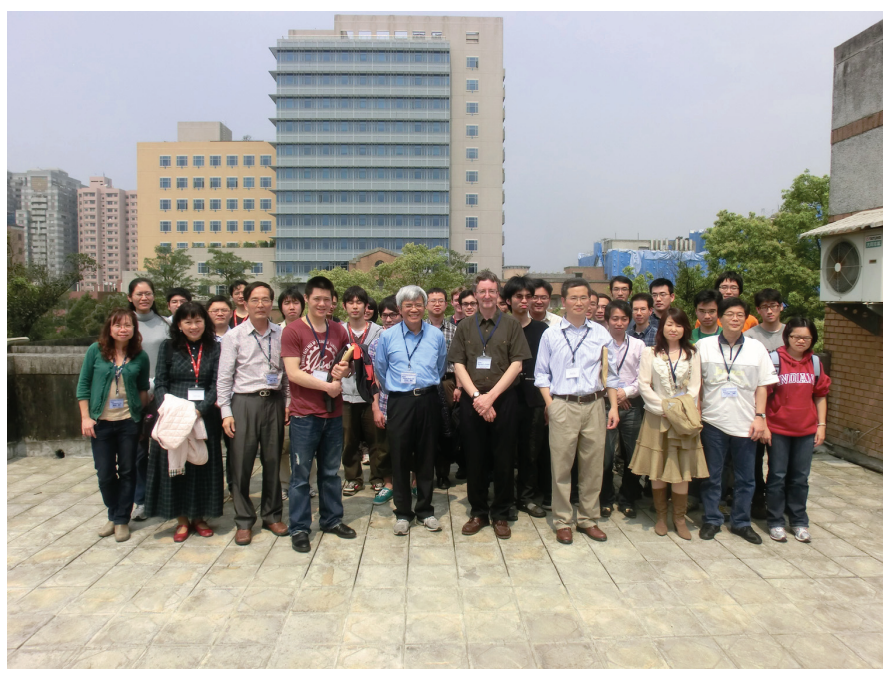

Integrable systems and geometric PDEs

- Invariance of Gromov-Witten theory under simple flops, by Y. Iwao Y.-P. Lee, H.-W. Lin, and C.-L. Wang, in Crelle 2012.

\section{Fu Szu-Nien Award in Mathematics}

In order to promote mathematics researches in Taiwan as well as to encourage outstanding mathematical contributions, NTU together with TIMS, has established the "Fu Szu-Nien Award in Mathematics" in 2011. The laureate will receive an award of NTD 1,000,000 each year for 3 consecutive years. During this period, the laureate also will teach and do research on campus of NTU for at least two years.

In 2011, the committee of Fu Szu-Nien Award give the first Award to Mu-Tao Wang from Columbia University. Having made great achievements in the area of differential geometry and partial differential equations in the last 
decade. he is well-known internationally for his deep researches in the theory of mean curvature flow and General Relativity. He was the first to develop mean curvature flow in higher co-dimensions. He also collaborates with Shing-Tung Yau in introducing the right concept of quasi-local mass, thereby successfully answered one of the main questions in General Relativity. In 2011/2012 $\mathrm{Mu}$-Tao Wang was a visiting Chair Professor at NTU and gave an advanced course on Topics in General Relativity.

\section{Prospects at TIMS}

Research faculty at TIMS consists of senior members (academicians from the Academia Sinica), 3 NTU chair professors, 5 full professors, and 4 young assistant professors. The faculty together with their collaborating international team, is responsible for organizing the various research activities at TIMS. The National Science Council fully supports TIMS under its highest level Academic Summit Program and Science Vanguard Research Program. The junior mathematicians at TIMS selected from the best of younger generation in Taiwan, are working on the most important frontier research topics, and making continued progress.

\section{New Faculty since 2010}

\section{Wu-yen Chuang}

Chuang received his B.S. in Physics at NTHU in 1998 and Ph.D. in Physics at Stanford University in 2007. He was post-doctor at Rutgers University from 2007 to 2010. He then joined NTU as an assistant professor since 2010. Chuang's research interests are on string theory and its related mathematical theories in algebraic geometry. Currently he works on quiver sheaf theory, framed sheaves and surface operators, and moduli problem with wall-crossing phenomenon. Chuang had received the Kenda Foundation Golden-Jade Fellowship from 2010 to 2014.

\section{Chen-Yu Chi}

Chi received his B.S. in Mathematics at NTU in 2001 and Ph.D. in Mathematics at Harvard University in 2009. He was a Harvard Junior Fellow from 2009 to 2012. He then joined NTU as an assistant professor since 2012. Chi had received the Levenson Teaching Prize in 2007 and the New World Mathematics Award for Ph.D. thesis in 2010. Chi's research interests are on algebraic geometry, complex analytic geometry and differential geometry. Currently he works on analytic approaches toward algebraic geometry based on pseudo-norms on pluri-canonical spaces, and algebro-geometric methods in differential equations including the Toda systems. 